Original Research Paper

\title{
Computer Vision for Vulnerable Road Users using Machine Learning
}

\author{
${ }^{1}$ Devi Chilukuri, ${ }^{1}$ Sun Yi and ${ }^{2}$ Younho Seong \\ ${ }^{I}$ Department of Mechanical Engineering, North Carolina A\&T State University, Greensboro, USA \\ ${ }^{2}$ Department of Industrial and Systems Engineering, North Carolina A\&T State University, Greensboro, USA
}

\section{Article history}

Received: 04-02-2019

Revised: 02-03-2019

Accepted: 09-03-2019

Corresponding Author:

Devi Chilukuri

Department of Mechanical

Engineering, North Carolina

A\&T State University,

Greensboro, USA

Email: dchilukuri@aggies.ncat.edu

\begin{abstract}
Transportation has become a very important component for all ages in today's life. However, seniors often find difficulty in travelling by public transport or even by their own vehicles. Thus, they are considered as Vulnerable Road Users (VRUs). This research mainly concentrates on creating an application that helps the elderly mobile users in virtually screening of directions using GPS, tracking of sidewalks, identifying the traffic signals and other sign boards. MATLAB/Simulink serves as the main software used in developing this application. Images and other types of data are captured using sensors on Android devices. Then MATLAB is used to perform deep learning. Transfer learning is another machine learning technique where a model like AlexNet developed for one task is retrained to perform a second task. In this research, AlexNet is used to identify and classify different traffic warning signs in real-time. A detailed description of this method and the results are presented in this paper. Once the MATLAB-based program is developed, it can be converted into Java codes when needed. Using Android Studio, the code can be used in the application. VRUs with mobility impairments and vision deficiencies often find it difficult to use wheelchair on sidewalks. This paper also presents a sidewalk tracking system with a departure warning. In this research, Hough Transform is used to present the detection of sidewalk. This sidewalk tracking system can provide the user with essential information that can minimize the risk of an accident. This system can identify and track the present sidewalks. Any unintended departure towards the edge of sidewalks will be detected and notified to the user. Elaborated implementation of this system and results are presented in this paper. Re-routing the user when they approach the end of the sidewalk is left for future work. The application will further be developed to provide a voice navigation informing the departure warning, a traffic signal or a recognized sign board.
\end{abstract}

Keywords: Computer Vision, Machine Learning, MATLAB/SIMULINK, Vulnerable Road Users

\section{Introduction}

Augmented Reality (AR) is a rapidly growing technology, that uses virtual information with real-time environment. This technology is used here to support the elderly mobile users with mobility and independence. The main challenge in developing AR systems is how realistically they integrate augmentations into the real world. Augmented reality can be used to assist aged drivers and Vulnerable Road Users (VRUs) to reach their destination safely with ease. Elderly people usually suffer from vision deficiencies and mainly involve in running traffic lights. Ocular diseases such as macular degeneration, cataracts, glaucoma, diabetic retinopathy and retinitis pigmentosa could increase the risk of vehicle crash. AR systems can improve communication of elderly and people with such vision related deficiencies through sound, speech and/or vibration. 


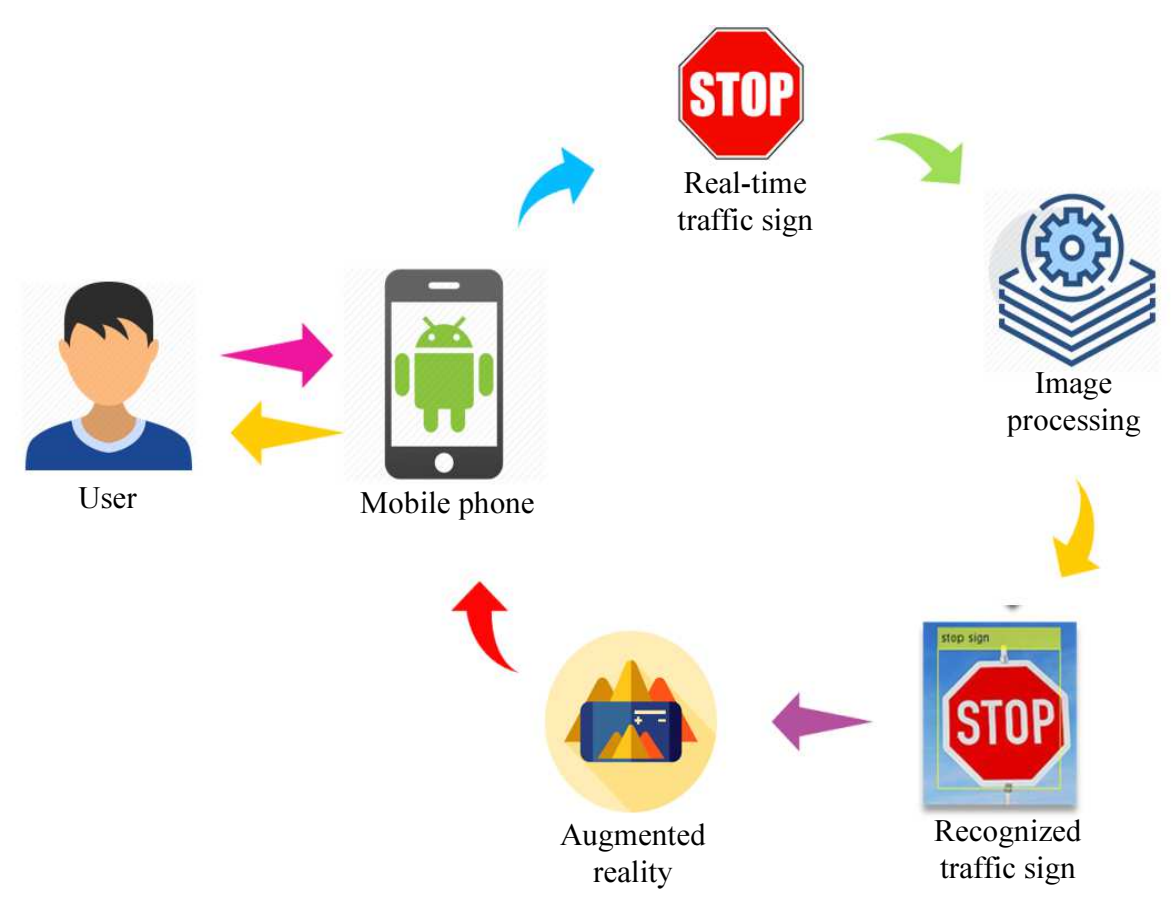

Fig. 1: The process flow involved in traffic sign recognition

MATLAB $^{\circledR}$ and Simulink $^{\circledR}$ provide a platform to explore images and develop computer vision algorithms. Images can be analyzed, through live acquisition or by postprocessing in MATLAB (MathWorks, 2017). It is helpful in performing Optical Character Recognition (OCR), face detection and recognition, edge detection, pattern recognition, feature extraction, template matching etc. by using computer vision techniques.

This research mainly concentrates on creating an application for elderly mobile users based on augmented reality. Android studio is used to develop this application. It has a strong editor tool for developing creative User Interface (UI). This mobile application is primarily being developed for Android devices. So, a Samsung Galaxy S8+ mobile phone is being used to test this application. The basic process flow involved in this app development is depicted in Fig. 1.

\section{Literature Review}

According to Hersh (2018) there are approximately 285 million people, who are visually impaired in this world. Among which, two-thirds are senior citizens of age 65 years and above. Mobility of these people require proper information regarding the direction of travel by avoiding obstacles and by moving in a straight line (Hersh, 2018). This research deals with the concerns mentioned above by tracking the sidewalks and detecting the obstacles blocking the way. He suggested the design considerations, interface design for developing a mobile application for mobility impaired (Hersh, 2018).

Quality of life in adults will be greatly affected by visual impairment. It can have a severe negative impact on their mobility. According to Nyman et al. (2012), older people with visual impairment often report impaired social interaction, minimum participation in daily activities, emotional stress and depressive symptoms. According to National Highway Traffic Safety Administration (NHTSA), 4743 pedestrians were killed in U.S in 2012 and older pedestrians amount to $20 \%$ of all pedestrian fatalities and up to $9 \%$ of all pedestrian injuries (Kim and Ulfarsson, 2018). It is also reported that most of the older pedestrian crashes occur at the intersections. Another research conducted on Older Road User (ORU) crashes in Australia, has yielded similar results (Koppel et al., 2018). Four main factors of risk are considered like roads and roadsides, road users, vehicles and speeds. Among them, the road user factor has experienced more crashes. The pedestrian environment should be improved, or they should be equipped with an assisting device to minimize the occurrence of accidents.

To improve the quality of life of the visually impaired and to provide them with safety and independence, researchers are coming up with new IT-based mobile assistive technologies. Many such innovations are reviewed and documented in (Hakobyan et al., 2013). Researchers are focusing more on the sighted guidance, as it is the most effective means of mobility assistance. 
In this process, teleassistance systems were developed in the past to provide remote guidance to the visually impaired by recording and transmitting the environmental information around the user through technology and by delivering the verbal instructions regarding directions to the user (Hakobyan et al., 2013). However, more efficient assistance systems were invented today to satisfy the visually impaired needs. Many prototypes for obstacle detection also were developed in the past, which involve cumbersome hardware like cameras attached to the chest (Jose et al., 2011), portable computers in the backpacks (Calder, 2010), sensor units at the front of the user's shoes (Zhang et al., 2010), feedback units on the arms (Shoval et al., 2003), ultrasonic sensors in the belts etc. (Hakobyan et al., 2013). Researchers faced many challenges to achieve an assistance system that incorporates everything a visually impaired user might require during navigation and yet make the device or assistive system easily portable.

Thorsten Völkel et al. (2008) discusses the problems faced by mobility impaired pedestrians due to current navigational systems available, as they do not provide adequate data for a pedestrian with mobility impairments but rather focuses on car navigation. Also, these navigational systems work to the benefit of pedestrians without any mobility restrictions, but most of the requirements are not fulfilled in the case of a pedestrian with mobility impairments. So, a multimodal annotation of geographical data technique is developed by the researchers to deal with the already stated issue (Völkel et al., 2008). To achieve this, a survey has been conducted on a group of visually impaired respondents including wheel-chair users, blind, people with temporary mobility restrictions and their requirements are considered. Although, there are many recent developments in providing navigational systems, to meet the needs of mobility impaired persons, they still consist of drawbacks due to inadequate information regarding the geographical data such as exact location of pavements and detecting cracks and holes (Völkel et al., 2008). This could overcome this limitation, as any cracks in the sidewalk can be detected using computer vision. A detailed description of this method is given in following sections.

Augmented reality has created a revolution in the field of computer vision. It is the rapidly developing technology that is being used in almost every research area today. Some wayfinding systems are also designed based on augmented reality to aid the visually impaired. Most of them are in the form of wearable devices such as smart glasses to avoid the struggle of concentrating on the mobile screen while traveling. The augmented reality navigation will be projected on the smart glasses and the user can see through it (Prandi et al., 2018). NAVIG augmented reality assistance system is another wayfinding system using wearables, which provides high precision geolocalization for visually impaired using satellite, images and other sensors (Katz et al., 2012). Another wearable prototype system designed for obstacle detection can be found in (Froneman et al., 2017). This system consists of ultrasonic sensors to detect obstacles and sends back the information through an array of vibration motors.

In addition to the various kinds of visual impairments, some elders also suffer from a reading disorder called Dyslexia. Persons with this disorder often face difficulty in reading the text present on any sign boards on the road. Roca et al. (2018) has conducted research on a group of adults with dyslexia and some Variable Message Signs (VMS) including text messages and pictograms were assessed to them. Results showed that the adults with dyslexia had trouble reading the VMS with text message on it and required much longer time to react when compared to normal adults. In this research, a deep learning neural network is used to detect and recognize the traffic warning signs and other sign boards which can be beneficial to adults with dyslexia. Also, Optical Character Recognition (OCR) can be implemented to recognize the text messages on the sign boards.

Visually impaired individuals face many challenges in indoor environments as well. To assist them in navigating independently inside the house, a real-time obstacle detection application is developed (Jafri and Khan, 2018). Some interviews are conducted with visually impaired individuals to make sure the application developed will meet their actual needs. The researchers have used the Project Tango Tablet Development Kit to develop this application. This system works with obstacle detection and generates feedback to the user through vibrations of the tablet. The distance of the obstacle is specified by the frequency of vibrations and if the distance between the user and the obstacle is less than or equal to $1 \mathrm{~m}$, speech-based feedback is provided to the user through open-ear Bluetooth-enabled bone conduction-earphones. This speech-feedback is achieved by using Android text-tospeech (TTS) and the inbuilt functions of the tablet are used for vibrations (Jafri and Khan, 2018).

\section{Methodology}

\section{Traffic Warning Sign Recognition}

Deep learning is a subset of machine learning technique. By making use of deep learning, data such as video, image, text or sound can be classified automatically (MathWorks, 2018). Deep learning uses multi-layered artificial neural networks to perform identification tasks such as speech recognition, object 
detection, language translation and face recognition as shown in Fig. 2. A standard Neural Network $(\mathrm{NN})$ is a computing system which consist of interconnected processors or neurons which process information by their response to external input. Input neurons are activated through sensors receiving signals from the environment. The signal is transmitted to other interconnected neurons. The connection between two neurons is called an edge and the signal at an edge is a real number. Some neurons may perform triggering actions to influence the environment (Schmidhuber, 2015). Deep learning is also used in advanced driver assistance systems. The term 'deep' is used to describe the multi-layered network. Transfer learning is another machine learning technique where a model like AlexNet developed for one task is retrained to perform a second task. AlexNet was trained to recognize 1000 objects with a dataset of 1.3 million images. However, smaller datasets can also be used to achieve accurate transfer learning (MathWorks, 2018).

In this research, Firstly, the sample images of the traffic warning signs are loaded and served as an image datastore. These images in the imageDatastore are labeled automatically based on the folder names and the data is stored as an ImageDatastore object. Large image data can be stored in these image datastores and it can efficiently read batches of images during training of a convolutional neural network. Secondly, the pretrained AlexNet neural network is loaded. AlexNet is trained to identify and classify images into 1000 object categories such as animals, vehicles and electronics etc. with a dataset of more than one million images (Moulder et al., 2017). For the new classification problem, the last three layers of the pretrained network are configured for seven classes based on different warning signs and fine-tuned. From this pretrained network all the layers are extracted except the last three. These layers are transferred to the new classification task. The last three layers are replaced with a fully connected layer, a softmax layer and a classification output layer. The options of the new fully connected layer are specified according to the new data.

The fully connected layer is set in such a way, so that it has the same size as the number of classes in the new data. WeightLearnRateFactor and BiasLearnRateFactor values of the fully connected layer can be increased so that the learning is faster in the new layers than in the transferred layers. The images in the image datastores are of different sizes but, the network requires input images of size $227 \times 227 \times 3$. To automatically resize the training images an augmented image datastore is used. Additional augmentation operations on the training images such as randomly flipping the training images along the vertical axis and randomly translating those up to 30 pixels horizontally and vertically can be specified. These data augmentation operations prevent the network from over fitting and helps memorizing the training images with exact details (Moulder et al., 2017). The network that consists of the transferred and new layers is trained. Finally, the validation images are classified and recognized using the fine-tuned network. Figure 3 shows some of the recognized traffic warning signs.

\section{Obstacle and Uneven Sidewalk Detection}

Independent navigation for individuals suffering from vision related deficiencies can be extremely difficult due to the inefficacy in recognizing and avoiding obstacles. An assistive technology should be able to locate nearby objects swiftly to avoid any accidents. Many computer vision techniques are being developed recently to emphasize certain classes of objects such as floors, walls, people and cars. In this research, the pre-trained AlexNet is used to detect the obstacles and uneven sidewalks as well and the results are shown in Fig. 4.

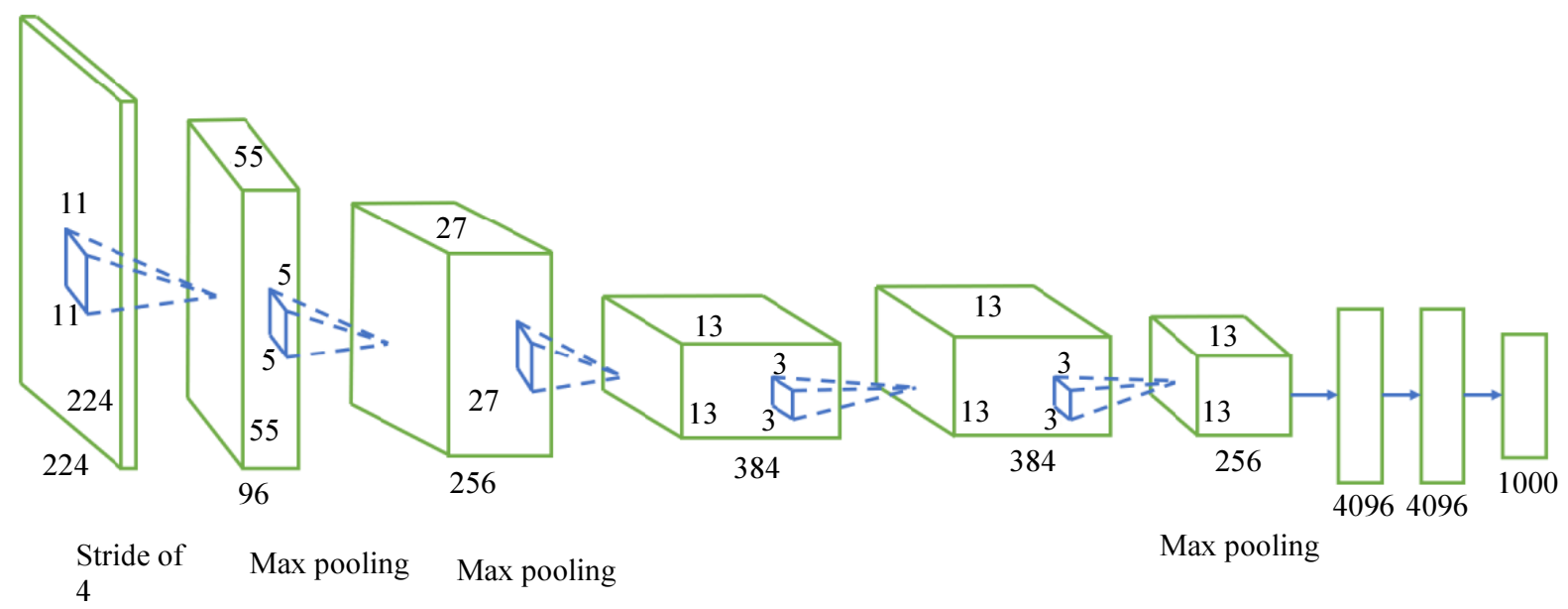

Fig. 2: The basic architecture of a neural network 

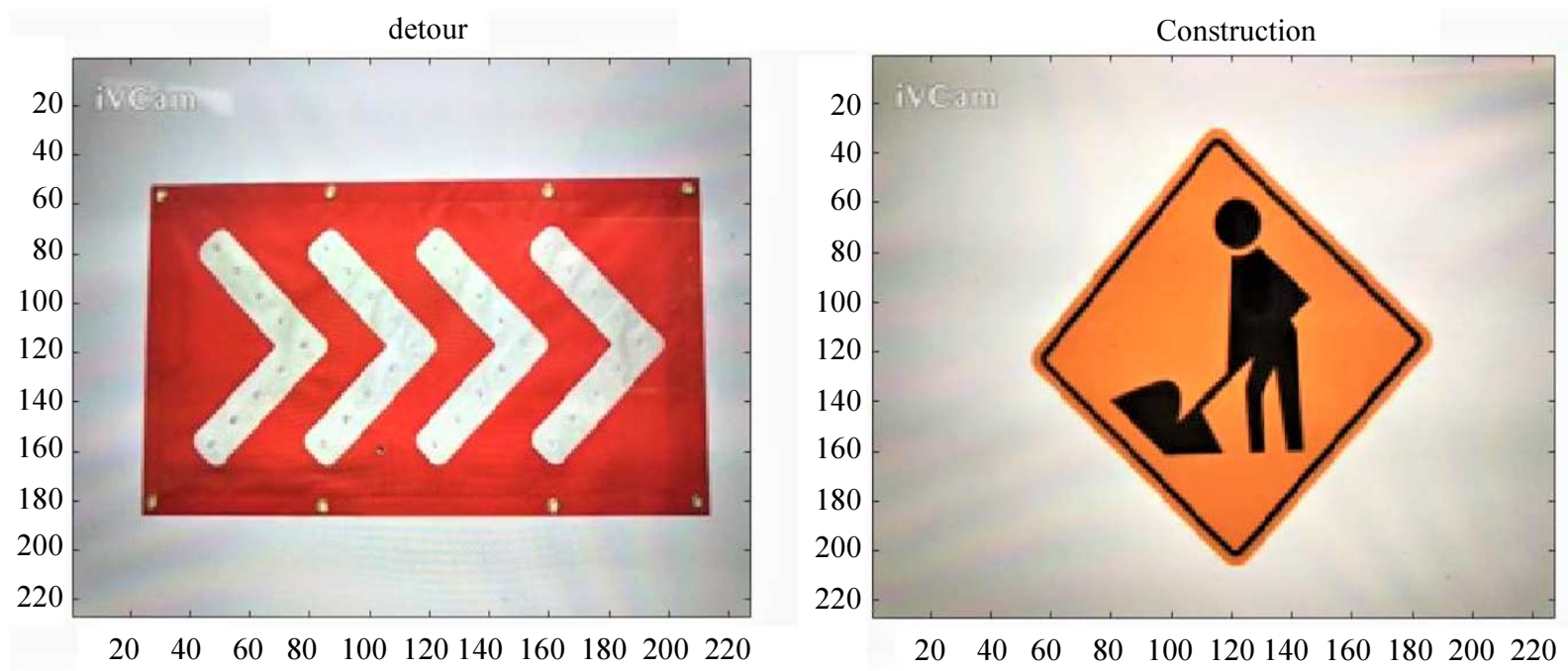

Fig. 3: Results showing the recognized detour and construction sign
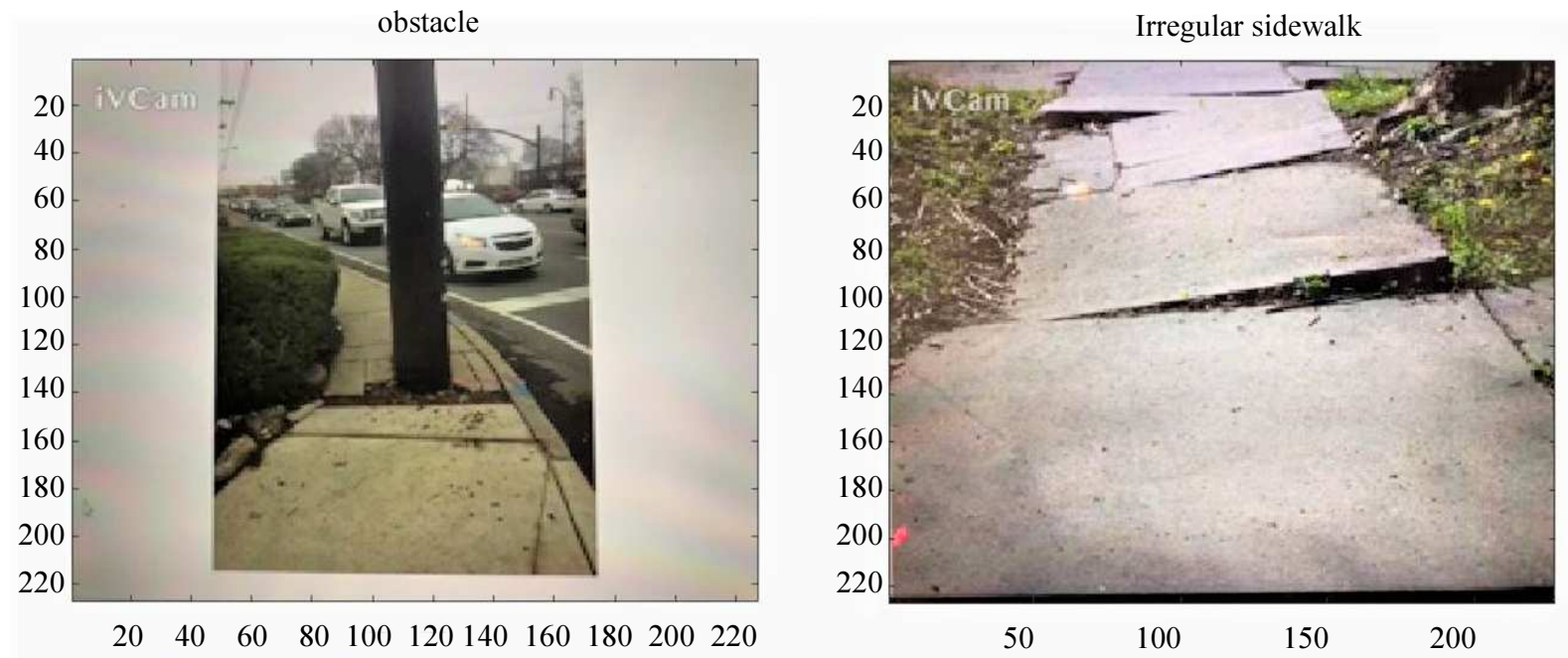

Fig. 4: Results showing the recognized obstacle and uneven sidewalk

\section{Sidewalk Tracking Based on Color Gradient}

In urban areas, sidewalks are mostly covered with green grass on either side. Hence, to detect the sidewalks in this case, an algorithm which works based on color gradient is used to detect and track the sidewalk edges. This algorithm performs in three main steps, which are explained below.

\section{Color Segmentation}

Firstly, the input image undergoes down-sampling process to reduce the computation. This is done by nearest-neighbor interpolation method, which means the average weight of nearest neighboring pixels is calculated and assigned to the missing pixels (Zhang et al., 2018). So, the input image is reduced by $50 \%$ (or by a resize factor of 2) from this process. Next, the image is converted from R' G'B' (normalized input of Red, Green and Blue) space to HSV space by using a color space conversion block. HSV stands for Hue, Saturation and Value, which provides the representation of image according to human visual perception (Kaur and Banga, 2013). The formula for converting $R^{\prime} G$ ' $B$ ' color space to HSV color space is given below in Equation 1: 


$$
\begin{aligned}
& H=\left\{\begin{array}{c}
\cos ^{-1} \frac{\frac{1}{2}\left[\left(\frac{R}{255}-\frac{G}{255}\right)+\left(\frac{R}{255}-\frac{B}{255}\right)\right]}{\sqrt{\left(\frac{R}{255}-\frac{G}{255}\right)^{2}+\left(\frac{R}{255}-\frac{B}{255}\right)\left(\frac{G}{255}-\frac{B}{255}\right)}}, \text { H is } 0-255 \\
\left(\frac{H}{255} * 360\right) \bmod 360, \text { H is } 0^{\circ}-360^{\circ}
\end{array}\right. \\
& S=\left\{\begin{aligned}
1-\frac{3}{\frac{R}{255}+\frac{G}{255}+\frac{B}{255}}\left(\min \left(\frac{R}{255}, \frac{G}{255}, \frac{B}{255}\right)\right) & \text { Sis } 0-255 \\
\frac{S}{255}, & \text { Sis } 0-1
\end{aligned}\right. \\
& V=\left\{\begin{array}{c}
\frac{1}{3}\left(\frac{R}{255}+\frac{G}{255}+\frac{B}{255}\right), \text { Vis } 0-255 \\
\frac{V}{255}, \text { Vis } 0-1
\end{array}\right.
\end{aligned}
$$

HSV color space provides robustness to lighting changes and removes the effect of shadows which are the major concerns while detecting the sidewalks. Morphological operations like erosion is performed on the image. It helps in removing the pixels from image boundaries, thus eliminating the disconnected line segments.

\section{Lane Detection}

The image is divided into two components: Left component, right component and the edges are detected in both the parts separately and simultaneously. In the pre-processing step, smoothing can be done to minimize any disturbances caused due to shadows of trees or buildings. This step helps in getting better accuracy while detecting edges (Aung and Zaw, 2014). Edge detection process is very common and most important in image processing, as it detects the boundaries of objects in images (MathWorks, 2017). Morphological operators like 2D-FIR filter and dilation filter are applied on the binary image to smooth the noise for better edge detection and to fill up the gaps. This works well even when there is a gradual change in the lighting conditions (Aung and Zaw, 2014). Once filters are applied and smoothing is done, Hough transform algorithm (Duda and Hart, 1971) is used to detect the sidewalk lane. This algorithm uses the following Equation (2):

$\rho=x \cos \theta+y \sin \theta$

where, ' $\rho(r h o)$ ' is the distance from the origin to the vector perpendicular to the line and ' $\theta$ (theta)' is the angle between $x$-axis and the vector.

Usually, the equation of straight line in Cartesian coordinate system is given as $y=m x+c$. In this case, Hough transform transforms the points in the Cartesian coordinate system to polar coordinate system by using the equation (Aung and Zaw, 2014; Mandlik and Deshmukh, 2016), to compute the parameter space matrix as shown in Fig. 5. The rows of this matrix represent the rho $(\rho)$ values and the columns represent theta $(\theta)$ values. The peak values in this matrix represent the potential straight lines in the image (Aung and Zaw, 2014). At the end of detection process, Rho $(\rho)$ values are up-sampled by 2 times, so that they get scaled back to the original resolution of the input image. Finally, Rho and Theta values are assigned to both the lanes.

\section{Lane Tracking}

During the tracking process, a lane must be detected in four continuous frames, to be considered as a valid lane. It can miss a maximum number of two frames, without getting marked as invalid. Since left and right lanes are being detected and tracked separately using different blocks, maximum of one lane will be found in the current frame. Once the valid left and right lanes are obtained from two blocks, the lanes are merged and displayed in one frame (MathWorks, 2017). Finally, the display block shows the tracking of valid sidewalk lanes as shown in Fig. 6. When both the lanes of the sidewalk are detected, then an arrow will appear indicating the direction of the sidewalk. If no lanes are detected, then a warning called "no sidewalk" is appeared on the screen.

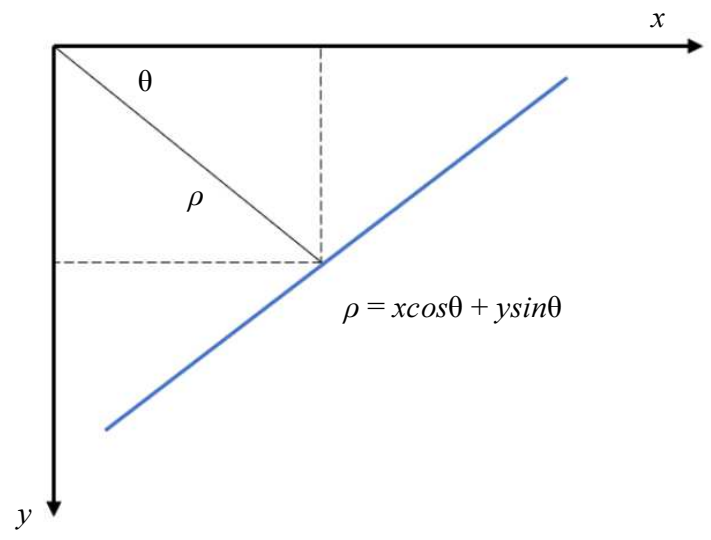

Fig. 5: Hough transform algorithm 


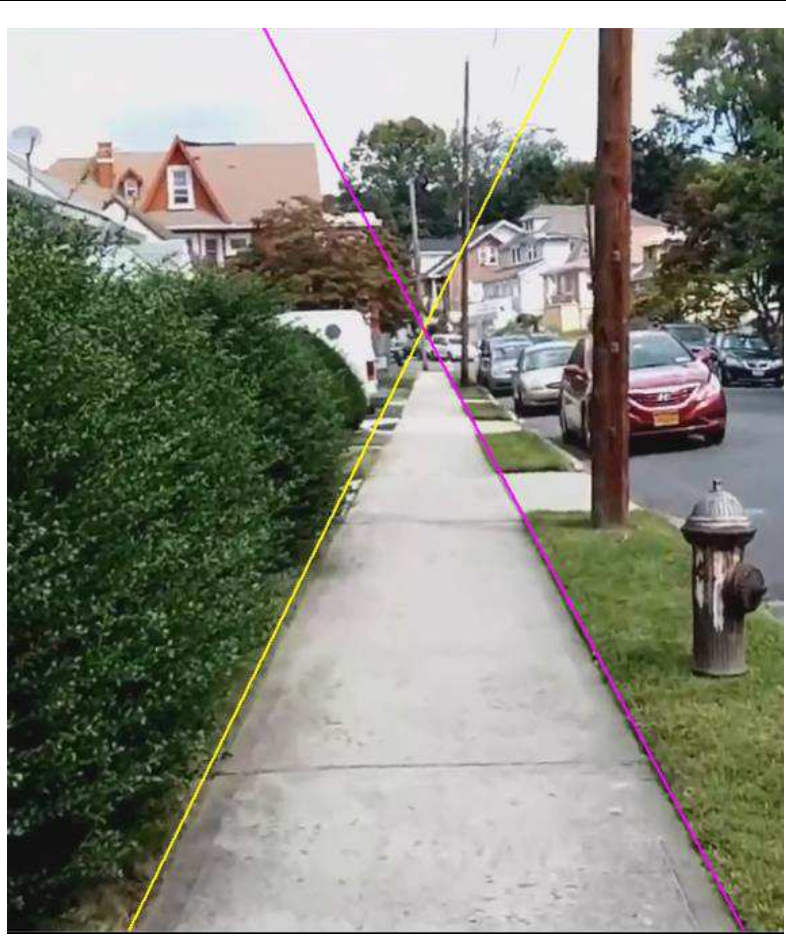

Fig. 6: Detected sidewalk using color gradient method

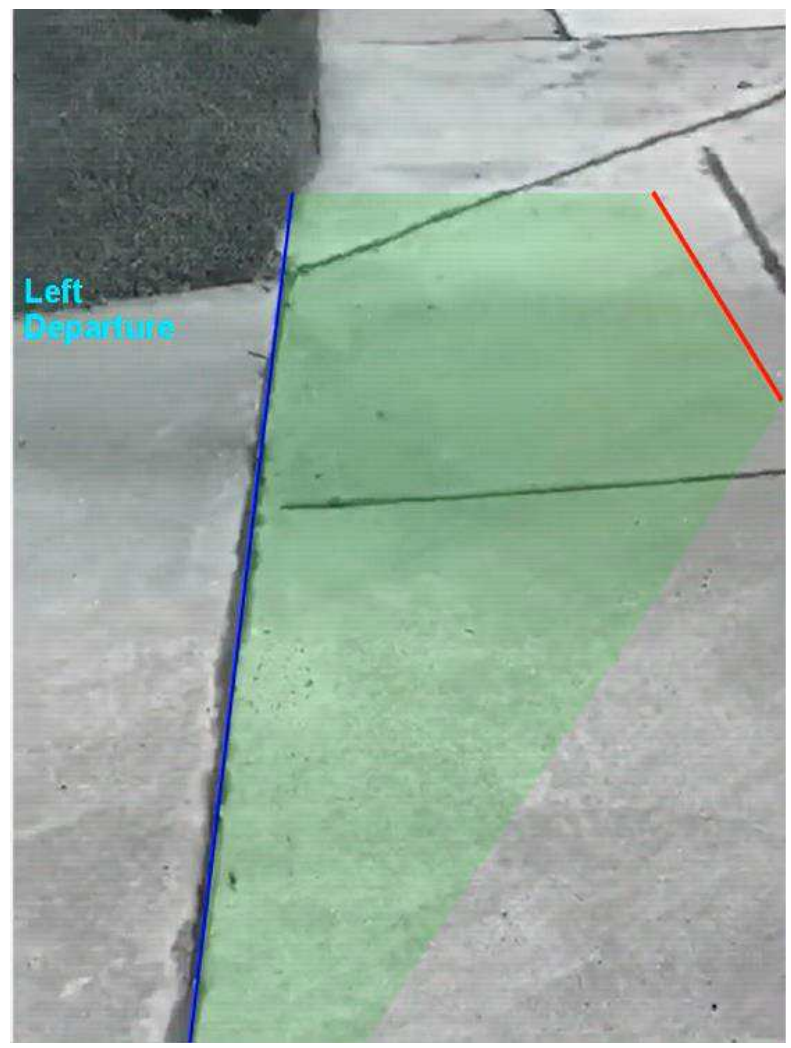

Fig. 7: (a) Tracking of sidewalk at the intersection. (b) Tracking of sidewalk at the intersection with a departure warning sign

\section{Sidewalk Tracking at Intersections with Departure} Warning System

A similar algorithm is used to detect the sidewalks at the intersection of two roads, where the sidewalk is usually not covered by the grass on both sides. As mentioned in the above section, a two-dimensional Finite Impulse Response filter (2D-FIR filter) is used to identify lane markers under various lighting conditions. This filter removes noise and the interference of shadows. The image is filtered first along the columns and then along the rows since, the applied filter is two dimensional. The overall luminance of the image is normalized by performing the auto thresholding operation. This converts the greyscale image from the input to binary image (Cho et al., 2014; Mani et al., 2010).

Hough transform is used to detect the straight lines which are described by theta and rho pairs. So, the inputs to the system consists of theta and rho values of lines and a reference image (Aung and Zaw, 2014). The lane markers in this case are defined on the basis of their angle with the reference image plane. These lane markers are tracked and matched using Kalman filter (Aung and Zaw, 2014). Sometimes, the edges of the sidewalk will be covered with grass or there can be some obstacles on the way and the edges might not be clearly visible. In such cases, the lane markers cannot be tracked properly. Kalman filter estimates the values of current state variables based on the previous data and thus the lane markers will be tracked continuously. Any movement towards these lane markers, activates the departure warning system. If the system detects the wheel-chair movement towards the left lane markers, a 'Left Departure' warning will be issued and similarly, if the wheel-chair moves across the right lane markers, a 'Right Departure' warning sign will be issued by the system as shown in the Fig. 7.

\section{Conclusion and Future Work}

In this paper, problems faced by vulnerable road users are addressed. The importance of developing a mobile application for elderly is discussed. The main focus of this application is to help senior citizens travel on roads/sidewalks safely without running traffic signals or traffic warning sign boards like stop, do not enter and yield. Sign boards along the roads like yield sign, construction sign, detour sign and few obstacles like a traffic cone is also detected and recognized in real-time. Sidewalks have been tracked and a departure warning sign is issued if the wheel-chair is moving towards the edge of the sidewalk. Along with, "No Sidewalk" warning is given when the user reaches the end of the sidewalk. This system is also able to detect and recognize uneven sidewalks. Re-routing the user after reaching the end of sidewalk is left for future work. The 
application will further be developed to provide a voice navigation informing the departure warning, a traffic signal or a recognized sign board.

\section{Acknowledgement}

The authors are thankful to Center for Advance Transportation Mobility for providing funds to pursue this research. We are also grateful to the North Carolina A\&T State University for providing the infrastructure.

\section{Author's Contributions}

Devi Chilukuri: Collected the data, conducted the experiment and obtained the results.

Sun Yi: Developed the theoretical framework and supervised the research.

Younho Seong: Designed the study of humanmachine interaction. Provided the critical revision of the manuscript.

\section{Ethics}

This article is an original research paper. There are no ethical issues that may arise after the publication of this manuscript.

\section{References}

Aung, T. and M.H. Zaw, 2014. Detection of lane departure using 2-D FIR and Hough transform. Int. J. Sci. Eng. Technol. Res., 3: 1302-1306.

Aung, T. and M.H. Zaw, 2014. Video based lane departure warning system using Houg transform. Proceedings of the International Conference on Advances in Engineering and Technology, Mar. 2930, Singapore, pp: 85-88.

Calder, D.J., 2010. Ecological solutions for the blind. Proceedings of the 4th IEEE International Conference on Digital Ecosystems and Technologies, Apr. 13-16, IEEE Xplore Press, Dubai, UAE, pp: 625-630. DOI: 10.1109/DEST.2010.5610585

Cho, A.N., C.M. New, H.M. Tun and M.Z. Oo, 2014. Design and simulation of vehicle lane tracking using MATLAB. Asian J. Eng. Technol., 2: 185-190.

Duda, R.O. and P.E. Hart, 1971. Use of the Hough trasformtion to detect lines and curves in pictures. Defense Technical Information Center.

Froneman, T., D.V.D. Heever and K. Dellimore, 2017. Development of a wearable support system to aid the visually impaired in independent mobilization and navigation. Proceedings of the 39th Annual International Conference of the IEEE Engineering in Medicine and Biology Society, Jul. 11-15, IEEE Xplore Press, Seogwipo, South Korea, pp: 783-786. DOI: 10.1109/EMBC.2017.8036941
Hakobyan, L., J. Lumsden, D. O'Sullivan and H. Bartlett, 2013. Mobile assistive technologies for the visually impaired. Surv. Ophthalmol., 58: 513-28. DOI: $10.1016 /$ j.survophthal.2012.10.004

Hersh, M.A., 2018. Mobility Technologies for Blind, Partially Sighted and Deafblind People: Design Issues. In: Mobility Visually Impaired People, Pissaloux, E. and R. Velazquez (Eds.), Springer, Cham, pp: 377-409.

Jafri, R. and M.M. Khan, 2018. User-centered design of a depth data based obstacle detection and avoidance system for the visually impaired. Human-Centric Comput. Inform. Sci., 8: 14-14. DOI: $10.1186 / \mathrm{s} 13673-018-0134-9$

Jose, J., M. Farrajota, J.M.F. Rodrigues and J.M.H. du Buf, 2011. The Smart vision local navigation aid for blind and visually impaired persons. Int. J. Digital Content Technol. Applic., 5: 362-375. DOI: $10.4156 /$ jdcta.vol5.issue5.40

Katz, B.F.G., S. Kammoun, G. Parseihian, O. Gutierrez and A. Brilhault et al., 2012. NAVIG: Augmented reality guidance system for the visually impaired. Virtual Reality, 16: 253-269. DOI: $10.1007 / \mathrm{s} 10055-012-0213-6$

Kaur, S. and D.V.K. Banga, 2013. Content based image retrieval: Survey and comparison between RGB and HSV model. Int. J. Eng. Trends Technol., 4: 575-579.

Kim, S. and G.F. Ulfarsson, 2018. Traffic safety in an aging society: Analysis of older pedestrian crashes. J. Transport. Safety Security, 2018: 1-10.

Koppel, S., L. Bugeja, D. Smith, A. Lamb and J. Dwyer et al., 2018. Understanding fatal older road user crash circumstances and risk factors. Traffic Inj. Prev., 19: S174-S176. DOI: $10.1080 / 15389588.2018 .1426911$

Mandlik, P.T. and P.A.B. Deshmukh, 2016. Image processing based lane departure warning system using Hough transform and Euclidean distance. Int. J. Res. Scientific Innovat., 3: 41-46.

Mani, J.R., N.D. Gangadhar and V.K. Reddy, 2010. A real-time video processing based driver assist system.

MathWorks, 2017. MATLAB and computer vision system toolbox release. The MathWorks, Inc., Natick, Massachusetts, United States.

MathWorks, 2018. Introducing deep learning with MATLAB. MathWorks.

Moulder, S., T. Sheridan, P. Cavallo and G. Rossini, 2017. Deep learning with MATLAB and multiple GPUs. MathWorks.

Nyman, S.R., B. Dibb, C.R. Victor and M.A. Gosney, 2012. Emotional well-being and adjustment to vision loss in later life: A meta-synthesis of qualitative studies. Disabil. Rehabil., 34: 971-81. DOI: $10.3109 / 09638288.2011 .626487$ 
Prandi, C., G. Delnevo and C. Ceccarini, 2018. On augmenting the experience of people with mobility impairments while exploring the city: A case study with wearable devices. Proceedings of the 15th IEEE Annual Consumer Communications and Networking Conference, Jan. 12-15, IEEE Xplore Press, Las Vegas, NV, USA, pp: 1-5. DOI: 10.1109/CCNC.2018.8319247

Roca, J., P. Tejero and B. Insa, 2018. Accident ahead? Difficulties of drivers with and without reading impairment recognising words and pictograms in variable message signs. Applied Ergon., 67: 83-90. DOI: $10.1016 /$ j.apergo.2017.09.013

Schmidhuber, J., 2015. Deep learning in neural networks: An overview. Neural Netw., 61: 85-117. DOI: 10.1016/j.neunet.2014.09.003

Shoval, S., I. Ulrich and J. Borenstein, 2003. NavBelt and the Guide-Cane [obstacle-avoidance systems for the blind and visually impaired]. IEEE Robot. Automat. Magazine, 10: 9-20.

DOI: 10.1109/MRA.2003.1191706
Völkel, T., R. Kühn and G. Weber, 2008. Mobility Impaired Pedestrians Are Not Cars: Requirements for the annotation of geographical data. Proceedings of the International Conference on Computers for Handicapped Persons, (CHP' 08), Springer, Berlin, pp: 1085-1092.

Zhang, H., D. Hernandez, Z. Su and B. Su, 2018. A low cost vision-based road-following system for mobile robots. Applied Sci., 8: 1635-1635.

DOI: 10.3390/app8091635

Zhang, J., C.W. Lip, S.K. Ong and A.Y.C. Nee, 2010. A multiple sensor-based shoe-mounted user interface designed for navigation systems for the visually impaired. Proceedings of the 5th International ICST Conference on Wireless Internet, Mar. 1-3, IEEE Xplore Press, Singapore, pp: 1-8. DOI: 10.4108/ICST.WICON2010.8516 